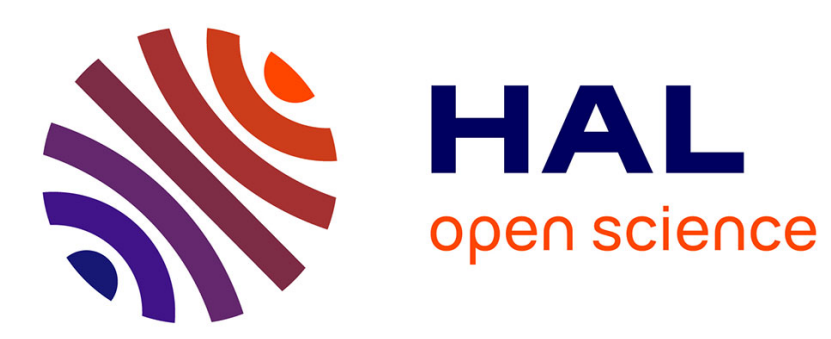

\title{
Combined-PSD and LIMM Measurements for Traps Characterization in BoPP Thin Films
}

\author{
Laurent Boudou, Laurent Berquez, G. Teyssedre
}

\section{To cite this version:}

Laurent Boudou, Laurent Berquez, G. Teyssedre. Combined-PSD and LIMM Measurements for Traps Characterization in BoPP Thin Films. IEEE Internat. Conf. on Dielectrics (ICD), Valencia, Spain, 5-9 July 2020., Jul 2020, Valencia, Spain. pp. 371-374. hal-03003191

\section{HAL Id: hal-03003191 \\ https://hal.science/hal-03003191}

Submitted on 13 Nov 2020

HAL is a multi-disciplinary open access archive for the deposit and dissemination of scientific research documents, whether they are published or not. The documents may come from teaching and research institutions in France or abroad, or from public or private research centers.
L'archive ouverte pluridisciplinaire HAL, est destinée au dépôt et à la diffusion de documents scientifiques de niveau recherche, publiés ou non, émanant des établissements d'enseignement et de recherche français ou étrangers, des laboratoires publics ou privés. 


\title{
Combined-PSD and LIMM Measurements for Traps Characterization in BoPP Thin Films
}

\author{
L. Boudou, L. Berquez and G. Teyssedre \\ LAPLACE, Université de Toulouse and CNRS, 118 Route de Narbonne, 31062 Toulouse Cedex 9, France.
}

\begin{abstract}
Understanding charge trapping phenomena in dielectrics requires a good description of the distribution in density, energetic levels, and if possible nature of the centers stabilizing charges. This topic is of great interest for many application fields as electronics or energy storage. Along this objective, fractionated-PSD (Photo-stimulated discharge) measurements, combined with space charge measurements using LIMM (Light Intensity Modulation Method) are developed in order to discriminate the origin of the different peaks observed in the PSD spectrum of Bi-oriented Polypropylene thin films.
\end{abstract}

\section{INTRODUCTION}

Charge trapping and transport phenomena in solid dielectrics are extensively studied since several decades. In particular, the study of charge accumulation is of great interest when considering its effects on electric field distortion and electrical properties modification. The space charge properties are closely linked to the characteristics of the traps (origin, density and depth in energy) present in the dielectric. Traps can be originated from physical or chemical defects [1], the corresponding depth in energy varying from low energies 0.1 $0.4 \mathrm{eV}$ (shallow traps) to energies higher than $1 \mathrm{eV}$ (deep traps). Different complementary methods, essentially with thermal or optical excitation, can be used to probe the traps. Thermally stimulated current and luminescence are well adapted to probe the shallow traps distribution [2], whereas Photo-Stimulated Discharge (PSD) is mostly used for traps in the range $1 \mathrm{eV}$ to more than $4 \mathrm{eV}$, depending on the light source characteristics [3, 4]. Recombination induced luminescence can also give information on deep traps acting as recombination centres [5]. Finally, space charge techniques can give the in-depth charge density profile and allow to follow the kinetics of the charge release [6].

The main advantage of PSD method is that it can be applied without any thermal effect that can affect the material microstructure and the charge stability. Nonetheless the interpretation of the PSD spectra is not straightforward. Actually, the discharge current can originate from different mechanisms: space charge detrapping, photoconduction effect, photo-emission from the electrode or from the dielectric material surface under test. It can also be perturbed by dipolar relaxations during measurement. In order to discriminate the origin of the different PSD peaks recorded during current measurements, the influence of different parameters can be tested: the geometry of the electrode facing the scanning light (full electrode, semi-transparent electrode, finger-like shape electrode), the nature of the metallic electrodes or the application of a small voltage during PSD measurements [4].

Space charge measurement can also give important information on the in-depth trapped charge distribution of previously charged sample, and allows to follow the space charge dynamics before and after light irradiation. For example, Mellinger et al [3] studied the space charge profiles, using piezoelectrically generated pressure step (PPS) method, of a gold-coated PTFE film corona-charged at $200{ }^{\circ} \mathrm{C}$ before and after irradiation at $240 \mathrm{~nm}$. After irradiation at $240 \mathrm{~nm}$, part of the charge was removed from the bulk of the polymer. The authors also point out the possibility of detrapping of near-surface charges, without experimental validation, because the PPS method cannot distinguish between near-surface charges and influence charges on the metal electrodes.

In this work, combined LIMM/PSD measurements are performed using the same apparatus offering the advantage to switch from one measurement to another without any sample manipulation. The first results obtained on Bi-oriented Polypropylene (BoPP) thin film are presented and discussed.

\section{METhODOLOGY}

\section{A. Combined PSD/LIMM apparatus}

Fig. 1 shows the diagram of the circuit for combined PSD/LIMM apparatus developed for this study. The $\mathrm{K}$ component allows to switch from PSD to LIMM and reciprocally, without any sample manipulation.

We used a fingered electrode as front electrode (facing the light during PSD/LIMM measurements) and full electrode as back electrode, connected either to an ammeter for PSD measurements or to a lock-in amplifier for LIMM measurements.

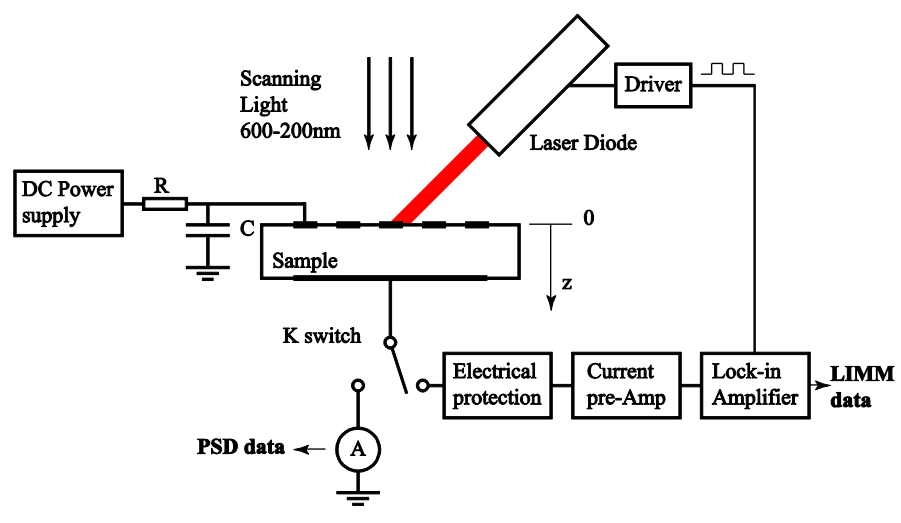

Fig. 1. Combined PSD/LIMM circuit diagram 
For PSD measurement, the monochromatic light (from $600 \mathrm{~nm}$ to $200 \mathrm{~nm}$ ) is produced by a $150 \mathrm{~W}$ Xenon arc lamp associated with a monochromator equipped with a $300 \mathrm{~nm}$ blaze grating $(1200 \mathrm{~g} / \mathrm{mm})$ as detailed in a previous communication [7]. The incident irradiation angle is normal to the sample surface and the lighted area is about $8 \mathrm{~cm}^{2}$.

For LIMM measurement, an intensity-modulated laser diode is used to heat locally the sample surface. The laser hits the sample surface on a "finger" of the fingered electrode (spot size about $2 \mathrm{~mm}$ in diameter) with an incident angle of approximatively $45^{\circ}$. The interaction between the produced thermal wave and the trapped charge allows to extract space charge density profiles from measured pyroelectric current, temperature profile modeling and mathematical treatment. The basic principle of the method, the theory and details on the setup can be found in [8].

The front electrode is grounded during the PSD and LIMM measurement and all the experiments were performed in air atmosphere and at room temperature.

\section{B. Material}

The sample under study is a $18 \mu \mathrm{m}$-thick BoPP, with rough surfaces to promote impregnation of capacitors. It was provided by Kopafilm, Germany. Test samples are discs of 8 $\mathrm{cm}$ in diameter, with gold electrodes sputtered on each face. For the electrode facing to the scanning light or to the laser diode (termed as the front electrode), we used a fingered electrode having a diameter of $4 \mathrm{~cm}$ with a thickness of $50 \mathrm{~nm}$, and fingers width of $4 \mathrm{~mm}$ [7]. For the electrode connected to the ammeter or to the LIMM detection system (termed back electrode), we used a circular electrode of $4 \mathrm{~cm}$ diameter and $50 \mathrm{~nm}$ thickness.

\section{Experimental protocol}

The experimental protocol used is represented in Fig. 2. Before the polarization step, blank PSD and LIMM spectra are recorded (PSD0 and LIMM0). Then the sample is polarized at a constant voltage of $3 \mathrm{kV}$, corresponding to an electric field of $167 \mathrm{kV} / \mathrm{mm}$, during $30 \mathrm{~min}$. After the polarization step, a first partial PSD measurement is performed by light scanning from long wavelength $(600 \mathrm{~nm})$ to an intermediate wavelength $(350 \mathrm{~nm})$, i.e. in-between the two PSD peaks previously reported for BoPP (400 $\mathrm{nm}$ and $220 \mathrm{~nm}$ ) [7].

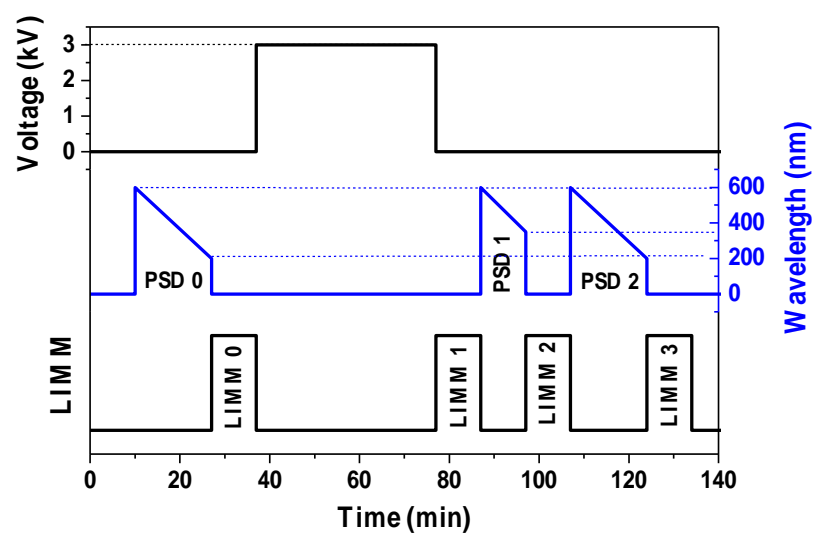

Fig. 2. Experimental protocol
The second PSD measurement is performed by scanning over the entire wavelength range from 600 to $200 \mathrm{~nm}$. LIMM recordings are performed at different periods during the protocol to follow the space charge evolution.

\section{RESULTS AND DISCUSSIONS}

\section{A. PSD/LIMM spectra}

The PSD spectrum recorded on the fresh sample is not totally flat. Two small current peaks are detected at $395 \mathrm{~nm}$ and $225 \mathrm{~nm}$, with amplitude lower than $1 \mathrm{pA}$ (Fig. 3).

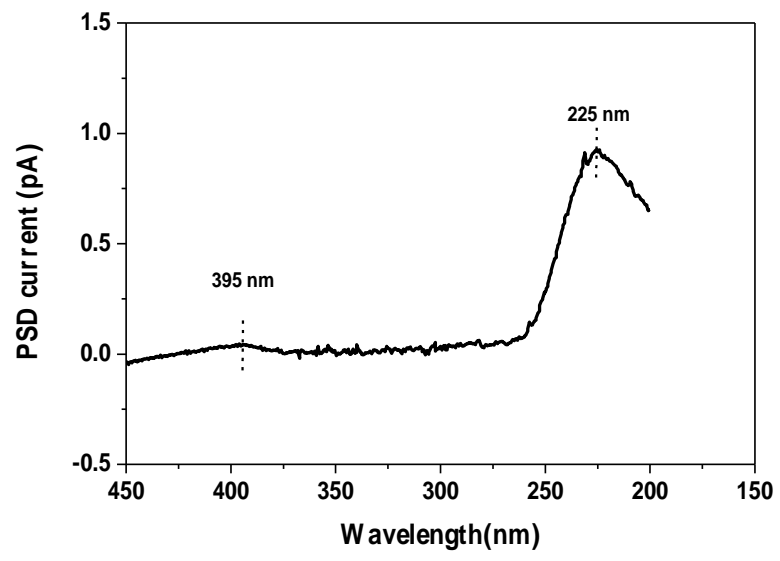

Fig. 3. Blank PSD spectrum recorded before polarization

The LIMM measurement performed before the voltage application (LIMM0 in Fig. 2) shows very low currents in the tested frequency range, corresponding to a negligible density of trapped space charge.

On Fig.4 are represented the PSD spectra obtained from partial (PSD1) and full (PSD2) light scanning. A first peak with a maximum located at $397 \mathrm{~nm}$ is recorded in both cases. This peak is relatively broad (spreading from $480 \mathrm{~nm}$ to 360 $\mathrm{nm}$ with a FWHM of $50 \mathrm{~nm}$ ) and does not correspond to known absorption band in BoPP investigated herein [9]. Either it can correspond to additives that could efficiently trap charges, or possibly to localized states formed under the effect of space charge [10].

A second peak is recorded in the wavelength range 260-200 $\mathrm{nm}$. The mathematical deconvolution (insert of Fig. 4) of this peak shows two contributions, located at 229 and $204 \mathrm{~nm}$, in agreement with earlier work [3]. These two contributions have to be correlated with the peaks observed in the absorption spectrum [9] of BoPP, that shows an absorption maximum at $200 \mathrm{~nm}$ and a first shoulder at $230 \mathrm{~nm}$, typical of $\pi \rightarrow \pi^{*}$ transitions in $\mathrm{C}=\mathrm{C}$ double bonds. A second shoulder at $280 \mathrm{~nm}$ is rather due to the $n \rightarrow \pi^{*}$ transition in carbonyl compounds. These two wavelengths (230 and $280 \mathrm{~nm}$ ) are those providing maximum photoluminescence in polyolefins [9].

Fig. 5 shows the charge density profiles obtained from LIMM measurements performed before PSD1, after PSD1 and after PSD2. After the polarization step and before PSD1 measurement, positive charges are accumulated near the $\mathrm{Au} / \mathrm{BoPP}$ interface (anode), corresponding to homocharges. 


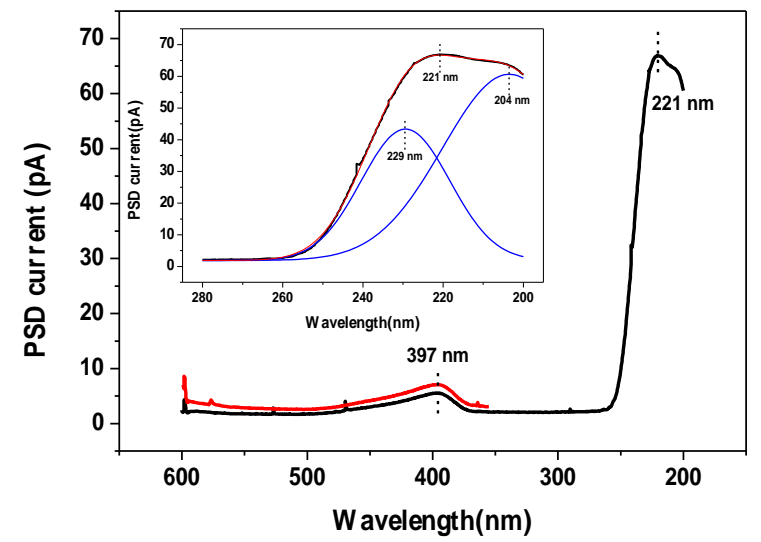

Fig. 4. PSD1 (red line) and PSD2 (black line) spectra after charging under $3 \mathrm{kV}$ for $30 \mathrm{~min}$. Insert: PSD2 spectrum in the range $280-200 \mathrm{~nm}$ showing the mathematical deconvolution

Furthermore, a negative charge peak appears within the bulk (between 2 and $7 \mu \mathrm{m}$ ) but we don't know at this stage if it corresponds to real negative trapped charges or to an artifact due to the LIMM signal processing. Finally, probing the trapped charges near the back electrode is difficult using LIMM. One can assume that homocharges, that is to say negative charges, can be accumulated at the cathode during the polarization step. If we consider the first $2 \mu \mathrm{m}$, corresponding to a depth range where the LIMM sensibility is highest, charge density is maximum after voltage application, then it decreases after the PSD1 and PSD2 scans are performed. Even though the difference between LIMM1 and LMM2 profiles shown on Fig 5 is weak, the trend is a decrease of the charge density when sample is lighted from 600 to $350 \mathrm{~nm}$ (PSD1) In addition, the LIMM3 profile performed after complete scanning (PSD2) also shows a decrease of charge density compared to LIMM2 profile.

According to these first combined PSD/LIMM results, charge detrapping can be one of the mechanisms responsible for the $395 \mathrm{~nm}$-peak recorded during PSD measurement.

Concerning the double PSD peak located in the 260-200 nm range, optical detrapping is not excluded, but other phenomena like photo-emission from the metallic electrode can be at play. Actually, by changing the metal nature of the front electrode, Ma et al [4] have shown a direct correlation between the work function of the metal and the cut-off wavelength of the peak observed in the $200-300 \mathrm{~nm}$ range for BoPP. The authors suggest that this peak results from electron emission via photoelectric effect from the front finger electrode when a local strong electric field exists.

Furthermore, during PSD measurement, charges can be detrapped and can move over short distances, depending on the internal electric field. The charge detrapping and transport can lead to a recordable current during PSD measurements, but the changes induced on the charge density profile are not necessary visible on LIMM results, because of the sensitivity and resolution of the method limited to $1 \mu \mathrm{m}$.

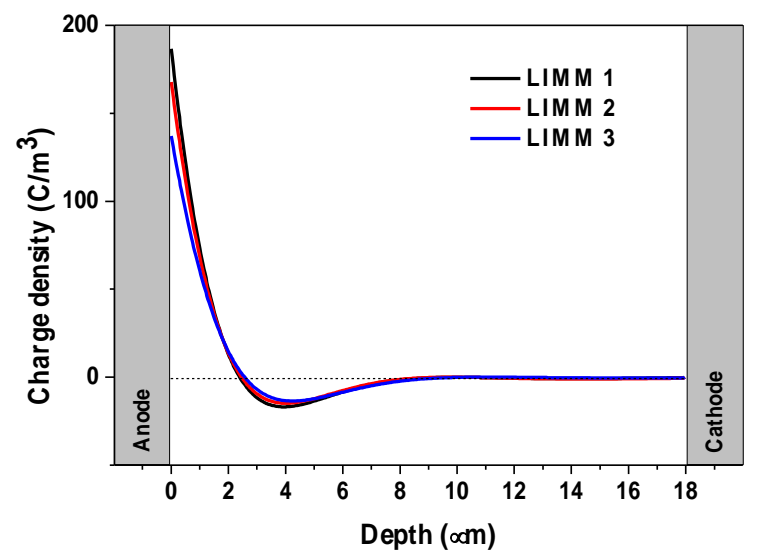

Fig. 5. Charge density profiles extracted from LIMM measurements performed at different time of the experimental protocol described in Fig. 2

One way to increase the transport of detrapped charge is to apply a small driving voltage during PSD measurement. Positive voltage (on top electrode) should drive positive charges in the bulk direction. Negative voltage should drive positive charges from bulk to the metal/polymer interface.

Another point to consider concerns the fingered electrode used as front electrode. It can first induce a field enhancement du to edge effect and as a consequence, charge injection and trapping at the edge of the fingers of the top electrode can be increased [4]. Besides, the fingered electrode plays a great role when considering the area (or volume) of the polymer material probed when LIMM and PSD are performed. A gold layer of $50 \mathrm{~nm}$ of thickness cannot be considered as transparent to the light: the transmission spectrum in the visible-UV range has to be considered for power generation and for qualitative analysis of the PSD spectrum. Another difficulty is that LIMM measurement probe the space charge trapped under the gold metallization and in a small area $\left(\approx 3 \mathrm{~mm}^{2}\right)$, corresponding to the spot size of the laser diode. Therefore, PSD and LIMM measurements do not probe exactly the same part of the polymer surface of the BoPP sample.

In conclusion, the interpretation of the origin of the different PSD peaks of BoPP is not trivial. The feasibility of fractionated PSD combined with LIMM has been demonstrated, which will help in the interpretation of the peak wavelength positions, but further investigations are needed.

\section{B. PSD transient current}

Measuring the current decay as a function of time under fixed wavelength is another way of analyzing the discharge phenomenon. Fig. 6 shows the transient photo-discharge current recorded at a fixed wavelength of $225 \mathrm{~nm}$ during $5 \mathrm{~h}$, by step of $1 \mathrm{~h}$, on a BoPP sample previously polarized at $3 \mathrm{kV}$ for $30 \mathrm{~min}$. LIMM measurements have been performed at different times during the decay and the obtained space charge density profiles are plotted in Fig. 7. During LIMM measurements, which last $10 \mathrm{~min}$ each for scanning frequencies from $10 \mathrm{~Hz}$ to $10 \mathrm{kHz}$, the $225 \mathrm{~nm}$-light irradiation is cut. 


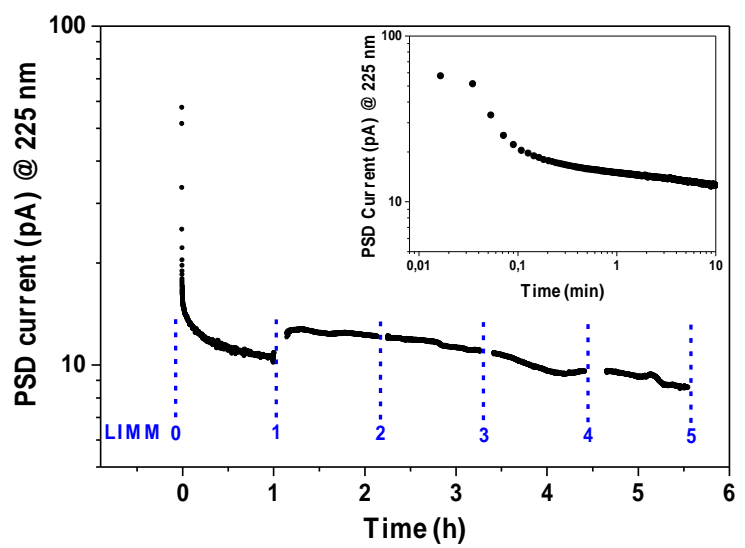

Fig. 6. Transient PSD current under continuous illumination at $225 \mathrm{~nm}$. Sample previously polarized under $3 \mathrm{kV}$ for $30 \mathrm{~min}$ Insert: zoom on the first $10 \mathrm{~min}$ of the transient current.

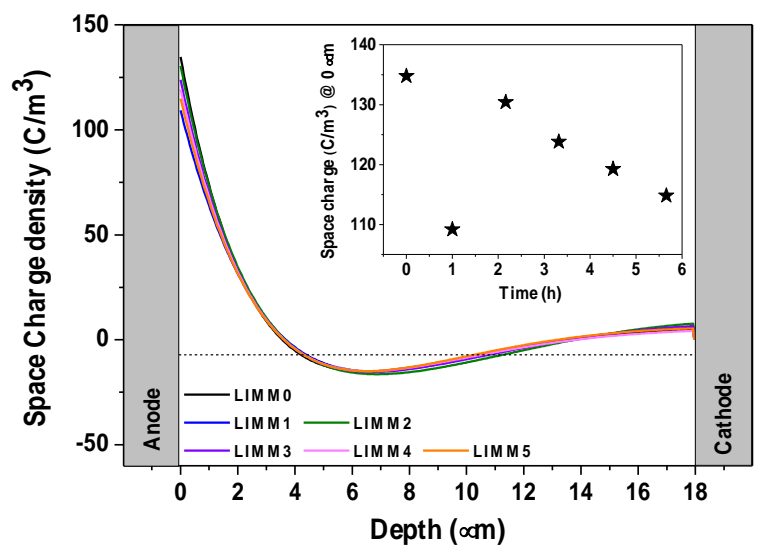

Fig. 7. Charge density profiles extracted from LIMM measurements. Insert: Evolution of charge density at the cathode/BoPP interface versus time

The first space charge profile (LIMM0) shows positive charge accumulation near the anode, in agreement with results presented in section III.A. The PSD current quickly decreases from $60 \mathrm{pA}$ to around $12 \mathrm{pA}$ during the first minute, before a much slower PSD current decrease is observed (cf. insert of Fig. 6). LIMM1 performed after $1 \mathrm{~h}$ of discharge shows a small decrease of the trapped charge at the anode (insert of Fig. 7) compared to LIMM0 (before PSD). After LIMM1 and when $225 \mathrm{~nm}$-light irradiation is turned on again, the recorded PSD current is slightly higher (12.3 pA) when compared to the current recorded after $1 \mathrm{~h}$ of discharge (10.5 pA) (Fig. 6). The next space charge profiles recorded and numbered from 2 to 5 in Fig. 7 show a slight decrease of the charge density at the anode/BoPP interface. The corresponding PSD current recorded during $1 \mathrm{~h}$ between each LIMM measurement shows a slow decrease and a non-monotonous shape of the decay.

One can assume that discharge current under long term illumination in the UV-range is due to several mechanisms in competition: photoemission from the electrode, detrapping of charge, etc. However, LIMM profiles are very similar and the difference of charge density at the anode/BoPP interface is weak. The relation between the light power reaching the material, the light absorbed locally and the efficiency of the light to detrap charges is far from being at reach.
The obtained results need to be confirmed by further investigation on samples polarized at higher field or during longer time, in order to have higher density of trapped charge, or charged by other means as electron gun. The transient discharge behavior has also to be investigated at a fixed wavelength of $395 \mathrm{~nm}, 229 \mathrm{~nm}$ and $204 \mathrm{~nm}$, corresponding to the maximum peak position shown in Fig. 4. Work has also to be achieved on LIMM improvement in order to get information on trapped charge at the rear electrode.

\section{CONCLUSION}

This work is a first attempt to perform combined LIMM/PSD measurements using the same apparatus offering the advantage to switch from one measurement to another without any sample manipulation. The results obtained on a $18 \mu \mathrm{m}$-thick BoPP film show three PSD peaks $(395,229$ and $204 \mathrm{~nm}$ ) and positive charge accumulation at the anode/BoPP interface is revealed by LIMM measurements. These results show also the difficulties and the necessary improvement of the combined LIMM/PSD method in order to correctly interpret the PSD spectra and space charge dynamics in BoPP thin films.

\section{ACKNOWLEDGMENT}

The research was carried with support from the IDEX Actions Thématiques Stratégiques - ATS 2015 of University of Toulouse, projet SEPHIR (2016-066-CIF-D-DRVD).

\section{REFERENCES}

[1] G. Teyssedre and C. Laurent, "Charge transport modeling in insulating polymers: From molecular to macroscopic scale," IEEE Trans. Dielectr. Electr. Insul., vol. 12, pp. 857-875, 2005.

[2] J. Van Turnhout, "Thermally stimulated discharge of electrets," Chap 3, pp. 81-215, in Electrets: Topics in Applied Physics, vol. 33, Ed. G. M. Sessler, Springer-Verlag, Berlin 1980.

[3] A. Mellinger, F. Camacho Gonzalez, and R. Gerhard-Multhaupt, "Photostimulated discharge in electret polymers: an alternative approach for investigating deep traps," IEEE Trans. Dielectr. Electr. Insul., vol. 11, pp. 218-226, 2004.

[4] P. Ma, Y. Zhang, S Holé, F. Zheng, M. Gua, and Z. An, "Analysis and interpretation of photo-stimulated discharge spectrum for polypropylene films under different electric and geometrical conditions," $J$. Electrostatics, vol. 84, p.128, 2016.

[5] G. Teyssedre, L. Cisse, C. Laurent, F. Massines, and P. Tiemblo, "Spectral analysis of optical emission due to isothermal charge recombination in polyolefins", IEEE Trans. Dielectr. Electr. Insul., vol. 5, pp. 527-535, 1998.

[6] L.A. Dissado, O. Paris, T. Ditchi, C. Alquie, and J. Lewiner, "Space charge Injection and extraction in high divergent fields," Proc. IEEE Conf. Electr. Insul. Dielectr. Phenomena (CEIDP), pp. 23-26, 1999.

[7] L. Boudou, F. Zheng and G. Teyssedre, "Photo- stimulated discharge current measurements on biaxially oriented polypropylene thin films (BOPP)", Proc. 12th ICPADM Conference, pp.722-725, 2018.

[8] A. Velazquez-Salazar, L. Berquez, and D. Marty-Dessus, "Towards space charge measurements by (F)LIMM under DC electric field", Proc. IEEE Internat. Conf. on Dielectrics (ICD), vol. 1, pp. 223-226, 2016.

[9] B. Qiao, G. Teyssedre and C. Laurent, "Field- and electron beaminduced luminescence phenomena in polypropylene thin films", Proc. 11th ICPADM Conference Sydney, Australia, pp. 196-199, 2015.

[10] C. B. Duke, "Optical absorption due to space-charge-induced localized states", Phys. Rev., vol. 159, p.632, 1967. 